\title{
La vigilancia en la obra de Philip K. Dick: Identidades, deseos y conflictos sociales
}

\section{Surveillance in the work of Philip K. Dick: Identities, desires and social conflicts}

Nelson Arteaga Botello

FLACSO, México

Resumen: Se analiza cómo la vigilancia en la obra de Philip K. Dick permite que actores e instituciones: $a$ ) construyan identidades, $b$ ) gestionen deseos y c) establezcan campos de disputas y conflictos de poder. La vigilancia aparece a veces como un mecanismo que sirve para la dominación y el control social, y en otras ocasiones como un dispositivo de resistencia que crea espacios de autonomía social. Por tanto, la vigilancia puede ser usada con fines civiles e inciviles. Los rostros de la vigilancia en Dick permiten entender como ésta se desarrolla en sociedades democráticas y diferenciadas, al contrario de la metáfora del "Gran Hermano" orwelliano desarrollada en un contexto donde predomina una ideología y un régimen político totalitario.

Palabras clave: mecanismos de vigilancia, control social, resistencia política, autonomía social.

Abstract: The article examines how surveillance, in Philip K. Dick's work, allows actors and institutions: a) to construct identities, b) to manage desires, and $c$ ) to establish areas of disputes and conflicts of power. Surveillance sometimes appears as a mechanism that serves as domination and social control, and at other times as a device of resistance that creates spaces of resistance and social autonomy. Therefore, surveillance can be used for civil and anti-civil purposes. The faces of vigilance in Dick's work allow us to understand how it develops in democratic and differ- 
entiated societies, unlike the metaphor of the Orwellian "Big Brother" developed in a context where an ideology and a totalitarian political regime prevail.

Keywords: Surveillance mechanisms, Social control, Political resistance, Social autonomy.

\section{Introducción}

La destrucción de la libertad y la autonomía individual tiene su drama más acabado en la sociedad híper-vigilada que dibuja George Orwell en 1984. La fuerza narrativa de esta historia es de tal magnitud que cuando alguien quiere enfatizar los peligros de la vigilancia en nuestras ciudades, el Internet, o las redes sociales, invoca el fantasma del "Gran Hermano" -ese personaje siniestro que monitoreaba las actividades de los ciudadanos del imaginado territorio de Oceanía-. Si bien otras novelas como Nosotros (Yevgeny Zamyantin), Himno (Ayn Rand), Un mundo feliz (Aldous Huxley), Fahrenheit 451 (Ray Bradbury), Justicia Facial (Leslie Poles Hartley), La fuga de Logan (William F. Nolan y George Clayton Johnson) o Un día perfecto (Ira Levin), logran dibujar atmósferas opresivas, no lo hacen al nivel de la novela de Orwell. Sin embargo, la metáfora del "Gran Hermano" tiene un costo: oscurece de alguna manera la compresión de los escenarios contemporáneos de vigilancia como proceso en el que se recolecta información de forma sistemática con el fin de influir en el comportamiento de grupos y personas (Nellis, 2009; Lyon, 1994). Como sugiere Marx (2009), uno de los problemas centrales de 1984 es que las distintas formas de vigilancia que ahí se despliegan se hacen acompañar de una ideología y un régimen político totalitario -un contexto político que comparten también el conjunto de novelas distópicas escritas en la misma época-, cuando hoy en día se puede observar 
que la vigilancia se expande y consolida en el conjunto de las sociedades democráticas. ${ }^{1}$

Autores como Philip K. Dick (1928-1982) lograron comprender que la vigilancia no necesariamente era una característica de los totalitarismos, también podía estar presente en las democracias. En este sentido, Dick planteó de forma acertada que la vigilancia tendería a desarrollarse en cualquier sociedad independientemente de su régimen político. Incluso para él, la vigilancia no era una actividad exclusivamente en manos del Estado o el gobierno, en sus historias es una práctica que despliegan distintos actores -a veces de forma más poderosa y efectiva que las propias autoridades gubernamentales- que van desde bandas criminales, corporaciones privadas, bancos, grupos terroristas y personas comunes y corrientes (Arteaga, 2015). En la medida en que la vigilancia tiene como fin modificar o mantener el comportamiento de los individuos en la sociedad (Lyon, 1994), aparece como un recurso ampliamente distribuido entre personajes e instituciones. Es por tanto un mecanismo de dominio y control social, pero también de resistencia frente al poder público y privado (Jameson, 2007). Así, los escenarios que describe Dick en sus novelas se alejan de alguna manera de la literatura distópica forjada en el contexto de los totalitarismos europeos del siglo XX y durante la Guerra Fría (Barceló, 2015). Un factor que quizás explica la atracción que tiene la industria fil-

${ }^{1}$ Estas ficciones que dibujan sociedades totalitarias comparten por lo menos cuatro elementos. En primer lugar, describen sociedades que han vivido o viven intensos y largos periodos de guerra. En segundo lugar, y debido a los escenarios bélicos, el Estado se encarga de proveer a su población de los satisfactores mínimos de subsistencia y, en esa medida, tiene la capacidad de decidir cómo deben vivir sus ciudadanos y durante cuanto tiempo. En tercer lugar, el Estado despliega un plan de homogenización social a partir de una ideología política, un modelo científico o una doctrina religiosa. Finalmente, ponen en marcha algún tipo de sistema socio-técnico de vigilancia (Arteaga, 2014). 
mográfica por llevar las novelas y cuentos de Dick al cine, así como la aceptación que estas producciones tienen entre el público. ${ }^{2}$

La presencia de distintos y variados dispositivos de vigilancia en la literatura de Dick puede atribuirse en parte, como sugiere Link (2010), a la consolidación de diferentes rutinas de vigilancia impulsadas, particularmente entre los ańos sesenta y setenta, por la Oficina Federal de Investigación y la Agencia Central de Inteligencia hacia la población de los Estados Unidos. Rutinas a las que fue sometido el propio Dick (Philmus, 1991). ${ }^{3}$ Sin embargo, es necesario reconocer que el escritor norteamericano veía que la vigilancia de alguna manera acompañaba la vida del hombre desde los primeros años de su historia. Dick (2005) afirmaba que los humanos habían desarrollado desde tiempos ancestrales un cierto estado de paranoia permanente como mecanismo de defensa ante la vigilancia de los animales predadores. Dicha paranoia era para

${ }^{2}$ Algunos de los filmes inspirados en su obra son Blade Runner (1982, EEUU, dir. Ridley Scott), basada en la novela ¿Sueñan los androides con ovejas eléctricas? publicada en 1968; Una Mirada en la Oscuridad [Scanner Darkly] (2006, EEUU, dir. Richard Linklater), inspirada en la novela del mismo nombre; Vengador del Futuro [Total Recall] (1990, EEUU, dir. Paul Verhoeven), que retomó el cuento "Podemos recordarlo por usted al por mayor" (1966); Screamers (1995, Canadá, dir. Christian Duguay), basada también en un cuento titulado "La segunda variedad" (1953); El Impostor [Impostor] (2001, EEUU, dir. Gary Fleder), inspirada en la novela corta del mismo nombre (1953); Minority Report (2002, EEUU, dir. Steven Spielberg), tomada del relato del mismo nombre publicado en 1956; Next (2007, EEUU, dir. Lee Tamahori), que apareció publicado como una historia breve bajo el título "The Golden Manen" en 1954; Paycheck (2003, EEUU dir. John Woo) inspirada en el relato corto "La paga" de 1953; TheAdjustment Bureau (2011, EEUU, dir. George Nolfi), basada en el relato Adjustment Team de 1954, y por último, Radio Free Albemuth (2010, EEUU, dir. John Alan Simon), inspirada en la novela del mismo nombre publicada de forma póstuma en 1985.

${ }^{3}$ A esto hay que sumar el hecho de que Dick tenía una personalidad con fuertes componentes paranoicos, lo cual acentuaba su obsesión por los mecanismos de vigilancia (Carrere, 2004). 
el novelista la respuesta apropiada al reconocimiento de que hay fuerzas amenazantes que se ciernen sobre los individuos. Según Sutin (1989), la diferencia que Dick encontraba entre el pasado y el presente era que la vigilancia contemporánea generaba la desafortunada sensación de que no se podía controlar a los actores y las instituciones que la operaban. La tecnología era responsable en gran parte de ello (Hayles, 1997). Ese sentimiento de constante amenaza había empezado a destruir, a decir del propio Dick, tanto la identidad que los individuos habían forjado de sí mismos (Mackey, 1988; Vizcarra, 2011) como la esfera de lo privado. Para Dick, según Williams (1986), parecía que ya no había nada secreto en la medida en que todo era prácticamente público.

Para algunos analistas, la vigilancia es un factor relevante en la obra de Dick porque genera la información que proporciona el "metabolismo del mundo moderno" y modela la manera en cómo los individuos se ven a sí mismos y a la sociedad (Lee y Sauter, 2000). Paradójicamente se ha puesto más atención en abordar otros temas de su obra, dejando la vigilancia como un aspecto secundario. Hay un mayor interés, por ejemplo, en comprender cómo Dick abordó cuestiones relativas a la epistemología, la naturaleza de la realidad, el sentido de lo humano, la entropía social y de los objetos, la guerra y los poderes políticos, la evolución humana, el problema de Dios y la tecnología (Link, 2010; Seed, 2011; Higgins, 2015; Wald, 2015; Sheldon, 2015; Best y Kellner, 2003; Hayles, 2005; García, 2006). El presente artículo se enfoca, por tanto, al análisis particular de la vigilancia en algunas novelas representativas del escritor norteamericano, en las que esta última aparece, a nuestro juicio, como un elemento clave en la conformación de la sociedad y de los individuos. Las novelas que se analizan son Lotería solar (1955), Simulacra (1964), ¿Sueñan los androides con ovejas eléctricas? (1968), Ubik (1969), Nuestros amigos de Frolik 
8 (1970), ${ }_{4}^{4}$ Fluyan mis lágrimas, dijo el policía (1974) y Una Mirada en la Oscuridad (1977). ${ }^{5}$

En este artículo interesa entender cómo la vigilancia se convierte en estas novelas en un dispositivo a través del cual distintos actores $a$ ) construyen identidades, $b$ ) gestionan deseos y $c$ ) establecen campos de disputas y conflictos de poder. Nuestro argumento es que las novelas analizadas dibujan un repertorio de categorías que revelan dinámicas sociales y personajes en situaciones típicas que cuestionan o idealizan una serie de valores, tales como el poder, la dominación, la libertad y la individualidad (Nellis, 2009). La vigilancia aparece a veces como un mecanismo que sirve para la dominación y el control social y en otras ocasiones como un dispositivo de resistencia que crea espacios de autonomía social frente a personas e instituciones. La vigilancia aparece así en algunos casos como un mecanismo con características civiles -con lo que se logra dar sustento a los ideales y principios como la libertad, la autonomía y la individualidad-y en otros casos con características inciviles -como una amenaza a esos principios e ideales-. Con este análisis se busca sugerir que la vigilancia para Dick, a diferencia de las distopías de corte más orwelliano, no es en todos los casos y en sí misma una amenaza, eso depende de los valores y acciones sociales que la movilizan.

El artículo abre con una revisión de conceptos básicos que se utilizan para analizar la manera en cómo las narraciones de ficción

${ }^{4}$ La versión en inglés de esta obra aparece bajo el título de Our Friends from Frolix 8; sin embargo, la traducción en español traduce el título como Nuestros amigos de Frolik 8. Utilizaremos este último título en la medida en que tomamos como referencia para este artículo la versión en castellano. También, las novelas aquí referidas son ediciones más recientes y están desglosadas en la bibliografía final.

${ }^{5}$ Cabe señalar que Lotería Solar fue escrita antes del asesinato del presidente Kennedy y las obras posteriores pertenecen a la era Nixon. Este último considerado como la bêtenoire de Dick. 
proyectan valores de libertad y autonomía a través de describir escenarios sociales opresivos. Tomando como principio de análisis la manera en cómo se aborda la vigilancia en los trabajos de Dick, se establece la estrategia metodológica y analítica del artículo. En los siguientes apartados se examinan, respectivamente, el papel de la vigilancia en la gestión de la identidad, los deseos y los conflictos sociales y políticos. En cada caso, se expone como Dick construye modelos de libertad y autonomía social frente a las estructuras jerárquicas de dominación. En un último apartado se reflexiona sobre esta paradoja de la vigilancia como un mecanismo de control y autonomía social. El artículo cierra con una serie de reflexiones que apuntan a destacar la importancia de la literatura de ficción para fortalecer el estudio de la vigilancia en las sociedades contemporáneas.

\section{Conceptos para el análisis}

Las narrativas literarias pueden entenderse como formas simbólicas que tejen códigos que reflejan los posibles modelos de libertad y autonomía social frente a relaciones autoritarias (Alexander, 2006). Siguiendo la perspectiva de la sociología cultural norteamericana, las novelas no son, como sugiere Habermas (1989), "sustitutos de relaciones con la realidad," que permiten a los lectores entrar en la acción de ficción como "un fondo de entrenamiento para la reflexión pública crítica" o como un "precursor literario de la esfera pública”, sino proyecciones e identificaciones estéticas, como dimensiones vitales en la formación de la opinión pública y del discurso civil (Alexander, 2006). Estos modelos expresan de forma problemática vivencias específicas. Las narrativas de ficción problematizan y estandarizan el marco de compresión de ciertos problemas sociales, proyectando sentimientos diversos, situaciones, tensiones y conflictos que muy difícilmente pueden materia- 
lizarse sino es a través de la ficción (Auotr, 2016). ${ }^{6}$ De esta forma, permiten entender nuestras sociedades actuales por juegos de comparación que se mueven en distintos tiempos: el pasado o el presente, en utopías o distopías (Marks, 2005; Karmmerer, 2012). Al problematizar temas como la libertad, la autonomía y la individualidad, se apela a la reflexión de valores en situaciones prácticas y concretas (Nussbaum, 1990). De igual forma, se puede ver a seres humanos en momentos donde se cuestionan principios morales y el propio sentido de lo humano (Rorty, 1989). Finalmente proyectan, a decir de Whitebrook (1996), situaciones donde los personajes se involucran en dilemas éticos con efectos políticos y sociales.

Para Palmer (2005), una de las aportaciones más significativas de Dick es precisamente que sus narrativas están llenas de encuentros, disputas, confrontaciones y traiciones en el que se cruzan, conviven y confrontan personajes tan distintos como plomeros, electricistas, mecánicos, policías, artistas, políticos y burócratas. En cada uno de esos encuentros los personajes se ven obligados a resolver problemas ligados a la construcción y comprensión de su identidad (¿quiénes son en realidad?) y de la sociedad que les rodea (¿qué es lo que pasa realmente en el mundo?) (Lem, 1975). La vigilancia es uno de esos ambientes, como sugiere Link (2010), donde Dick coloca los encuentros y contextos en el que interaccionan sus protagonistas. Por tanto, resulta un elemento clave a través del cual los distintos actores construyen su identidad, gestionan sus deseos y pueden influir de alguna manera en el orden político y social en el que viven. Este es, sin duda, uno de los grandes temas que Dick introduce al abordar la vigilancia y que lo distingue del trabajo de Orwell en 1984. La distopía de Orwell se dibuja como un gran panóptico en el que todos los aspectos de la vida humana son monitoreados por un poder central, en tanto que Dick sugie-

${ }^{6}$ Para profundizar en esta perspectiva de análisis de las ficciones literarias, ver: Williams (2001), Jameson (1989), Pia (1998) y Brooks (2005). 
re que la vigilancia no sólo recae en este último, sino que está en distintas partes: la vigilancia no sirve sólo para castigar a quien ha infringido una norma, sino que se ejerce como un dispositivo de justicia preventiva (Novell, 2009), en el que se puede castigar a las personas antes de que cometan supuestamente un delito (como se narra en el relato corto de Minority Report).

En las novelas ¿Sueñan los androides con ovejas eléctricas?, Fluyan mis lágrimas, dijo el policía y Una Mirada en la Oscuridad, se pude encontrar, por ejemplo, cómo se construyen las lógicas de identidad a través de la vigilancia y cómo los actores tratan de definirse, de distinta manera, frente a esas lógicas. En las novelas Nuestros amigos de Frolik 8 y Simulacra es posible observar, por otra parte, cómo las instituciones definen su actuación a partir del monitoreo de los deseos y apetitos, tanto personales como colectivos, no sólo con el objetivo de incrementar sus capacidades de control, sino para satisfacer las demandas cambiantes de la sociedad. Finalmente, en Lotería solar y Ubik, se puede apreciar la manera en que la vigilancia se construye como un proceso ligado a la competencia en la esfera política y social.

Con el fin de analizar precisamente esta relación de la vigilancia con la identidad, los deseos y la competencia por el poder, el trabajo desarrolló una metodología ligada a la perspectiva de la sociología cultural (Alexander, 2006). Esto significa que buscó resaltar cómo la vigilancia se inscribe en una serie de categorías dicotómicas en las que aparece operando a través de motivos y relaciones sociales inspiradas en principios civiles o inciviles. Siguiendo este orden de ideas, los textos seleccionados fueron leídos, en una primera instancia, observando cómo los actores usaban o apelaban a la vigilancia en función de valores ligados a la opresión social o para generar procesos de desigualdad e inequidad. En una segunda instancia los textos se leyeron en función de localizar cuándo los actores o las instituciones apelaban o utilizaban la vigilancia a 
partir de valores o acciones que respaldaban la libertad, autonomía y solidaridad. Esto permitió observar cómo las historias de Dick movilizan opiniones que presentan la vigilancia como un proceso que resalta a veces los ideales civiles y, en otros, como un proceso que amenaza esos mismos ideales.

Es necesario señalar que las referencias literarias que se consideraron para este artículo no representan el universo de los trabajos de Dick al respecto de la vigilancia -que alcanza más de una treintena de novelas y un gran número de cuentos y narraciones cortas-. Como sugiere Link (2010), no hay una sola manera de leer el trabajo de Dick, ni una obra que resuma sus ideas y obsesiones, sino que en cada una de sus historias se expresan los temas que fueron centrales en su obra. Aquí se han seleccionado las novelas que, a decir de los críticos más importantes de su obra (Booker, 1994; Link, 2010; Jameson, 2007; Link y Canavan, 2015; Freedman, 2000; Alfaraz, 2010), muestran a la vigilancia en su forma más elaborada. ${ }^{7}$

\section{Vigilancia y construcción de identidades}

La relación entre construcción de identidad y dispositivos de vigilancia en ciertas novelas de Dick está mediada por la forma en cómo se define el perfil de aquellas personas consideradas como un riesgo o amenaza al orden y la seguridad de la sociedad. Si bien es cierto en este perfil pueden caber individuos que han cometido

${ }^{7}$ La relevancia de la obra de Dick para la ciencia ficción estriba en que, como sugiere el crítico y novelista de ciencia ficción Stanislaw Lem (autor de la novela Soliaris, 1961), dibujó futuros en los que los dilemas abstractos y densos de la filosofía académica descienden a las calles, tales como la objetividad y subjetividad, y muestra cómo esclarecer estos dilemas resulta central para la supervivencia de sus personajes. Al traer estos dilemas a las calle, añade Link (2010), Dick proporciona un nuevo color a viejos temas filosóficos y teológicos que además le permiten criticar la cultura de su tiempo. 
algún tipo de crimen, delito o acto terrorista -establecido así por el orden legal de la sociedad que el autor imagina-, lo cierto es que a veces los personajes se ven obligados a mostrar constantemente quiénes son; si no lo hacen de forma satisfactoria, son considerados, por este hecho, como amenazas a la sociedad. Estos vínculos entre vigilancia e identidad se pueden encontrar en ¿Sueñan los androides con ovejas eléctricas?, una novela en la que los protagonistas se enfrentan constantemente al desafío de confirmar su humanidad ante autoridades gubernamentales o directivas empresariales. Mientras que en Fluyan mis lágrimas, dijo el policía, los personajes tienen que corroborar ante un amplio espectro de policías públicas y privadas su identidad e incluso sus distintas identidades -como trabajador, ciudadano, consumidor, entre otras tantas que puede poseer una persona. Finalmente, en Una Mirada en la Oscuridad, los protagonistas son sometidos a intensos procesos de identificación con el fin de mostrar en qué medida y cuándo han cometido algún tipo de crimen o acto ilegal. En cada una de estas novelas, sin embargo, la vigilancia puede evitarse y sabotearse, más aún, a veces la propia vigilancia genera tanta información que termina por volverse inútil para establecer la identidad de las personas y el perfil de su comportamiento.

Dick describe a la Tierra en ¿Sueñan los androides con ovejas eléctricas?, como un planeta que ha sido devastado por el uso de armas biológicas y radioactivas en lo se llamó la "Guerra Mundial Terminal". La contaminación radioactiva abarca buena parte del globo y las ciudades prácticamente resultan los únicos espacios seguros para habitar. La contaminación ha afectado también a los seres humanos. Quienes aún se encuentran sanos migran a Marte, mientras que aquellos con mutaciones se ven condenados a quedarse en la Tierra. La vigilancia de las autoridades está orientada a evitar que los humanos que han mutado logren salir del planeta. Sin embargo, hay un peligro mayor: que ingresen a la tierra 
los androides orgánicos que funcionan como esclavos en Marte. Uno de los dispositivos de vigilancia que garantizan una Tierra libre de androides, es el test de Voigt-Kampff. Éste relaciona una serie de respuestas a ciertas preguntas con las reacciones faciales del entrevistado, particularmente la dilatación capilar de su área facial. Con esto es posible detectar la vergüenza o el rubor ante un estímulo que sacude la moral. Los androides responden a los cuestionamientos morales, pero no se ruborizaban. Esta es la manera en cómo se puede distinguir al Otro no humano. Por ejemplo, según el test de Voigt-Kampff, los humanos responden con una desaprobación verbal y expresiones faciales de disgusto cuando se les pide su opinión sobre una escena en la que un niño es golpeado de forma salvaje para que ingiera sus alimentos. El entrevistado al identificarse con el niño proyecta un comportamiento empático que no está sujeto al control del entrevistado -como dice un personaje de la novela para mostrar la eficacia del test: la indignación "no se controla voluntariamente como la respiración" (Dick, 2012: 66). Los androides orgánicos desaprueban el hecho del niño maltratado, pero no hay ninguna emoción reflejada en sus rostros. La prueba es la clave para la identificación de los androides, por ello las instancias encargadas de cazarlos traen todo el tiempo consigo un maletín con los instrumentos para aplicar el test.

En Fluyan mis lágrimas, dijo el policía, el riesgo no está en la salida no autorizada de humanos de la tierra o el ingreso a ésta de androides orgánicos, sino en las actividades consideradas ilegales por un régimen político de carácter policial. Se disuaden y evitan las actividades consideradas ilegales a través de un amplio despliegue de mecanismos de control y vigilancia de los desplazamientos de la población. De esta forma Dick describe una sociedad en la que los edificios, calles, espacios comerciales y parques, cuentan con retenes que funcionan como puntos de revisión. Las ciudades están plagadas de puntos de detención en los que se identifica a 
las personas. La cantidad de retenes que un ciudadano debe superar en un recorrido normal por la ciudad puede llegar a treinta. En cada retén, los ciudadanos deben identificarse a través de una carta de identidad que contiene datos personales -algunas de ellas con micro transmisores para ser rastreados- que son verificados en tiempo real en los archivos correspondientes en las oficinas de la policía o la guardia nacional.

Si una persona no se identifica plenamente en un punto de control, corre el riesgo de ser ejecutada de inmediato, o ser enviada a los campos de trabajo forzado, bajo el argumento de que son "no-personas", "hombres invisibles" o "legalmente invisibles". Por ello, algunos ciudadanos, ante el temor de extraviar u olvidar sus cartas de identidad nacional, se tatúan su número de identidad. No obstante, esto no es suficiente, en la sociedad que dibuja Dick, se requieren otro tipo de cartas para realizar las más variadas actividades, como caminar a ciertas horas en la ciudad, conducir automóviles, consumir legalmente drogas o tener trabajo. En general, las cartas tienen fecha de caducidad - pueden durar horas, días, meses o años-. Además, la policía tiene la autoridad de instalar -a ciertos sospechosos de cometer algún tipo de delito- microtransmisores subcutáneos o electroencefalogramas que envían información a la central de la policía. Esta información se cruza con aquella que obtiene la propia policía de sus cámaras de vigilancia instaladas por toda la ciudad y que recogen y procesan imágenes en tres dimensiones.

Para la mayor parte de los críticos es, sin embargo, Una Mirada en la Oscuridad, donde Dick logra conectar de mejor manera los procesos de identificación y vigilancia. El escritor norteamericano describe en esta novela una sociedad donde la droga Sustancia $D^{8}$ se ha esparcido a lo ancho y largo de los Estados Unidos. Sus efectos son devastadores en la población, ya que inhabilitan a su

${ }^{8}$ 'D' hace referencia a Death, muerte en Inglés. 
usuario para realizar casi cualquier actividad. Los periodos de recuperación son lentos y las secuelas afectan de por vida el sistema nervioso. El problema es aún más agudo por el hecho de que la policía no sabe cuál es la fuente biológica, química y territorial de donde se obtiene dicha sustancia. Por tanto, la policía despliega un amplio espectro de dispositivos de vigilancia en el territorio nacional. Destacan sobre todo los holoescáneres -especie de cámaras vigilancia- que monitorean la actividad de las personas de manera sistemática y permanente. Las escenas y diálogos que capturan dichos escáneres son reproducidos en tercera dimensión en proyectores holográficos.

No obstante, el logro tecnológico más importante descrito en la novela es el "traje codificado" -el scramblesuit-, un aditamento que como un pijama cubre el cuerpo de quien lo utiliza, transformando de esta manera su identidad física: estatura, complexión, color de piel, rostro (incluida la nariz, boca, color de ojos). Modifica también la vestimenta en función de cálculos algorítmicos que generan un número incontable de combinaciones de pantalón, camisa, corbata, sombrero, zapatos y calcetines, que pueden llegar a repetirse sólo después de un ciclo de catorce años. El traje resguarda la identidad de los agentes de narcóticos cuando realizan su trabajo; además, un dispositivo electrónico en el traje permite la comunicación permanente con la central de policía. Desde el traje se puede tener acceso a información en "burbujas" -especie de pequeñas pantallas- sobre las personas que se está investigando. Este tipo de dispositivos hace ver la intervención telefónica y la implementación de micrófonos en la ropa o en los aparatos radiofónicos -que por su puesto también aparecen en la novela-como dispositivos de vigilancia poco sofisticados.

Como se puede observar, la vigilancia en las tres novelas aquí analizadas está orientada a la definición y localización de ciertas personas que se consideran un riesgo o amenaza social. Su ope- 
ración genera claros procesos de exclusión, estigmatización social y, en algunos casos, la detención y la muerte de los personajes. La vigilancia es caracterizada de esta manera como un instrumento que se mueve a partir de principios inciviles. Es una herramienta que mina la integridad de las personas y las vuelve vulnerables al control político. Sin embargo, eso no excluye que los protagonistas desarrollen mecanismos de resistencia, utilizando para ello los propios dispositivos de vigilancia en un sentido opuesto al establecido por las autoridades gubernamentales o al poder de las corporaciones privadas. Así, orientan de alguna manera la vigilancia en un sentido claramente civil. Por ejemplo, en ¿Sueñan los androides con ovejas eléctricas?, los protagonistas, no importa si son o no son androides orgánicos, deslegitiman en distintos momentos el uso del test Voigt-Kampff, sugiriendo a los policías que primero se apliquen a ellos mismos la prueba, lo que mina la legitimidad y uso de la prueba. Por otro lado, en Fluyan mis lágrimas, dijo el policía, pese a que los personajes de la novela afirman que "una vez que las autoridades se fijan en uno, jamás lo olvidan del todo" (Dick, 2015: 250), al pasar el tiempo, a decir de los propios protagonistas, "el aparato policial se convirtió en algo demasiado complicado como para amenazar a nadie"(265). Esto se debió particularmente dos procesos. El primer lugar, se generó un mercado ilegal de cartas de identidad -a veces alimentado por la propia policía- que hizo imposible distinguir entre las auténticas y las falsas. En segundo lugar, la cantidad de información producida por una vigilancia cada vez más compleja -orientada además a detectar las identificaciones falsas- acabó por volver inoperante el proceso de identificación de cualquier individuo.

Aunque ciertamente el desgaste de la vigilancia como medio de identificación y control social no necesariamente implica su abandono o su uso para fines civiles, sí genera la sensación de que la realidad se vuelve algo evanescente e inasequible, incluso socava 
la propia identidad de quien está vigilando. Esto queda planteado de alguna manera en Una Mirada en la Oscuridad. Los propios policías saben que ellos también están bajo vigilancia y se sienten amenazados a un punto tal que ni ellos mismos saben quiénes son en realidad. Como afirma el protagonista de la novela:

Si el escáner sólo ve oscuramente, como yo, estamos condenados, condenados otra vez, como siempre, y terminaremos igual, sabiendo muy poco y entendiendo mal lo poco que sabemos porque en estos días ni yo soy capaz de ver claramente, porque en estos días ni yo soy capaz de ver dentro de mí. Sólo veo tinieblas. Tinieblas fuera; tinieblas dentro. Espero, por el bien de todos, que los escáneres lo hagan mejor (Dick, 2006: 182).

Aquí, la vigilancia es considerada por quienes la operan como una herramienta incivil que los despersonaliza a ellos mismos. Los holoescáneres se convierten en objetos con atribuciones mágicas que nadie controla porque todos los usan de alguna manera, no son ya algo humano, son algo muerto que puede ver, "aunque no sean capaces de comprender: son nuestra cámara" (Dick, 2006: 183).

\section{La gestión de los deseos}

La vigilancia de los deseos sociales es otra de las aristas de la obra de Dick. En varias de sus obras plantea que el objetivo de este tipo de monitoreo es determinar, desde los aparatos gubernamentales o corporaciones empresariales, qué sienten las personas con el fin de responder a sus necesidades emocionales. No hay por tanto un grupo particular sobre el que se cierne la vigilancia, sino que ésta se dirige al conjunto de la población. Esta vigilancia busca amoldar las políticas gubernamentales y los productos en el mercado a las necesidades de ciudadanos y consumidores. Por supuesto, 
la gestión de las emociones desde el ámbito gubernamental tiene un límite. Cuando el cuestionamiento a la autoridad se transforma en rebeldía, la vigilancia opera sus mecanismos de confinamiento o asesinato de aquellos a quienes considera como una amenaza. Enfrentar este control político sólo puede llevarse a cabo a través de una vigilancia desplegada desde la sociedad, la cual permanece aún cuando el sistema de dominación se ha desmantelado. Esto es lo que sucede en Nuestros amigos de Frolik 8 y en Simulacra.

Dick describe en la primera novela un mundo que se encuentra gobernado por humanos mutantes -los "inusuales"-, que poseen una inteligencia superior y la capacidad de leer y, por tanto vigilar, la mente del resto de las personas. ${ }^{9}$ Su dominio lo ejercen sobre otros hombres menos dotados (llamados según sus capacidades, "sub-hombres", "hombres antiguos" o "nuevos hombres"). Usan para ello distintos dispositivos. Uno de ellos, "el gran oído", capaz de monitorear a gran escala y al mismo tiempo el pensamiento de los hombres de los grupos dominados. La vigilancia se ejerce con el fin de evitar cualquier acto de rebelión, pero también para satisfacer los distintos deseos de las personas y, por supuesto, algunas veces modificarlos. Así los deseos y aspiraciones de las personas no se suprimen en una primera instancia, se gestionan a favor del gobierno de los "inusuales". No obstante, cuando los dispositivos de vigilancia localizan a los opositores al régimen, estos son enviados a centros de reeducación en la Luna, si vuelven a reincidir, son remitidos a campos de exterminio.

${ }^{9}$ Como señala Link (2010), son figuras recurrentes en la obra de Dick estos mutantes que pueden leer la mente o predecir de alguna manera el futuro. A veces estos personajes se llaman "Precog". Aunque también Dick imagina dispositivos con el mismo objetivo, como en Los clanes de la luna Alfana (1964), donde describe la "foto potencial Afgom", la cual genera una imagen no de lo que están haciendo las personas, sino de lo que harán durante la siguiente media hora. 
Simulacra se desarrolla, por su parte, en un futuro donde los Estados Unidos y Europa (hasta los límites de la Alemania Occidental) forman un solo país: los Estados Unidos de Europa y América (EUEA). Esta nación es gobernada por un presidente androide. No obstante los ciudadanos ignoran esto y cada determinado periodo de tiempo eligen, a través de un proceso democrático, un nuevo presidente -el cual es otro androide. En realidad no hay una competencia de partidos ya que el Partido Republicano y Demócrata se han fusionado en el Partido Demócrata-Republicano. Si bien el presidente es sustituido periódicamente, la primera dama -Nicole Thibodeaux-, permanece siempre en su puesto. Ésta última es la cara visible de un consejo formado por líderes de corporaciones, quienes realmente controlan el país. Las elecciones tienen además otro objetivo: Nicole Thibodeaux y su consejo sondean los sentimientos y necesidades de la población, programando así al siguiente androide-presidente. Además, el gobierno cuenta con el dispositivo de Von Lessinger, una máquina del tiempo que sirve para vigilar e intervenir en el pasado. Así, la sociedad está constantemente vigilada tanto en el presente como en el pasado.

En estas dos novelas se aprecia cómo la vigilancia de los deseos garantiza la gestión y control de la población por parte de entidades gubernamentales y grupos corporativos en un sentido claramente incivil. Sin embargo, como todo sistema de dominación, hay quienes logran resistir a él y subvertirlo a través del uso de distintos dispositivos de vigilancia que responden a principios y valores civiles. En el caso de Nuestros amigos de Frolik 8 dicha resistencia adquiere el rostro de una revolución organizada, en los llamados "Dionisos de las alcantarillas", un grupo que difunde propaganda antigubernamental. El jefe de la revolución, Thors Provoni, es apoyado por Morgo Rahn Wilc, un ser del planeta Frolik 8, quien tiene la capacidad de sondear la mente de todos los habitantes de la tierra al mismo tiempo. Esto permite a los revolucionarios influir en el 
pensamiento de las personas contra la dominación de los "inusuales”. Gracias a las habilidades de Morgo Rahn Wilc, Thors Provoni interviene las transmisiones de la televisión -controlada en su totalidad por el gobierno- y los circuitos privados de comunicación de la policía, para enviar mensajes subversivos a la población. Sin embargo, los revolucionarios y su líder no logran minar la lógica de dominación basada en la gestión de los deseos del gobierno, por lo que Morgo Rahn Wilc decide extirpar la capacidad que tienen los "inusuales" de vigilar la mente. De esta manera, sugiere la novela, la libertad de los pobladores de la Tierra queda garantizada, paradójicamente, por un ser que tiene las mismas capacidades de vigilancia, o quizás mayores, que el gobierno de los “inusuales” que ha ayudado a destruir.

La idea de que la libertad no significa necesariamente el fin de la vigilancia se cristaliza también en Simulacra. Dick narra que aquellos que migran a Marte huyendo de la dominación de Nicole Thibodeaux y su consejo, ${ }^{10}$ adquieren de inmediato una "Familia Vecina" o "Famvec", un simulador que se comporta como un "buen vecino", pero que en realidad funciona como un sistema de vigilancia de los deseos. La función de esta familia de androides es monitorear los sentimientos de las familias humanas, recoger sus esperanzas ocultas, sus sueños y temores, de tal suerte que se convierten en dispositivos terapéuticos que garantizan la cordura de sus propietarios. Dick parece confirmar aquí el hecho de que la vigilancia puede ser benéfica en la medida en que permita a los seres humanos el conocer algo de sí mismos. Por tanto, es un mecanismo útil para potenciar los principios civiles y también garantizar hasta cierto punto el carácter humano de las personas.

${ }^{10}$ En el caso de esta novela, la única forma de escapar de la vigilancia de los Estados Unidos de Europa y América es huyendo a Marte. Aquellos que se quedan en la Tierra buscan, por su parte, evidenciar que el presidente del país es un androide. 


\section{Conflictos políticos y sociales}

La vigilancia no sólo sirve para identificar amenazas y gestionar deseos, es también una herramienta que sirve para sobrevivir en la esfera de la competencia política y para garantizar la autonomía individual y grupal frente a la injerencia de otras personas e instituciones. En el primer caso, Lotería solar es un buen ejemplo de cómo la vigilancia resulta un medio clave, incluso institucionalizado, para el funcionamiento del sistema político. En el segundo caso, Ubik resulta un ejemplo de cómo la vigilancia se pone abiertamente al servicio de cualquier individuo con miras a influir de alguna manera en su entorno y sobre otros individuos, pero también como un mecanismo para garantizar la autonomía frente a fuerzas externas. En ambas narraciones la vigilancia termina por convertirse en una moneda de cambio que forma parte de la vida diaria. Un bien al que se puede y debe acceder porque forma parte sustantiva de las relaciones sociales en ámbitos tan distintos como la vida política y personal.

El mundo que Dick describe en Lotería solar se caracteriza por ser una sociedad donde el azar funciona como el principio regulador de las relaciones y las instituciones sociales. La producción de bienes de consumo -casas, automóviles, muebles-, se lleva a cabo en grandes cantidades, pero sólo unos cuantos tienen acceso a ellos. La gran parte no se vende y se destruye. No obstante algunos objetos se sortean, de la misma manera que sucede con el prestigio y el poder. "La botella" es el "instrumento socializador del azar" (Dick, 2007: 7) que garantiza elegir a los gobernantes de forma aleatoria con el fin de evitar las batallas por el poder político. Para participar como elegible por "la botella" es necesario tener una Tarjeta-p, un carnet de identidad que da la "oportunidad entre seis mil millones de participar en la gran lotería, la remota posibilidad de acceder, mediante un salto inesperado de la botella, a la posi- 
ción de la clase número Uno" (8). Las tarjetas son codificadas para cada persona en el momento de su nacimiento, por lo que todos pueden participar como posibles opciones de "la botella" para llegar a la presidencia del mundo, o como se denomina en la novela, ser investido como el "Gran Presentador".

Buena parte de la población se dedica al cálculo de probabilidades con el fin de predecir si algún día podrán ganar algo -lo cual crea un comercio amplio de métodos que ayudan a predecir los patrones de aleatorios de la botella. Sin embargo, a diferencia de la rifa de los productos materiales en la sociedad -que se asignan sin discusión a quien resulta favorecido-, cuando alguien gana la presidencia, existe un reglamento que autoriza al presidente saliente a asesinar legítimamente al "Gran Presentador" entrante durante un plazo determinado de tiempo. Para garantizar que el nuevo elegido se mantenga en el poder, el Estado posee servidores públicos que lo resguardan, los llamados "Brigadas telepáticas del directorio": personas que vigilan los pensamientos del presidente saliente, sus colaboradores y aliados, para tratar de frenar los proyectos de conspiración contra el presidente entrante. Estas Brigadas pueden ser neutralizadas con robots humanoides manejados a distancia y por distintas personas con el fin de confundir a los telépatas. De esta forma, la vigilancia se convierte en un instrumento vital del sistema político: es el garante de la estabilidad política y de los acuerdos establecidos entre los grupos para respaldarlos procesos de selección democrática del presidente.

La vigilancia funciona también como un mecanismo de competencia y estabilidad social en Ubik. En esta novela, la sociedad del futuro se caracteriza porque los ciudadanos se vigilan unos a otros a través de la contratación de telépatas. La trama de la novela involucra dos corporaciones en competencia. Una dedicada a ofrecer sus servicios de vigilancia apoyada en los telépatas y la otra dedicada a vender servicios de bloqueo telepático. Ambas empresas 
utilizan los llamados pregcogs - personas que pueden predecir lo que sucederá en el futuro, pero sin la capacidad de cambiarlo- y los antipsi-quienes pueden incidir en el pasado pensando en él, sin que se den cuenta las personas. También utilizan antiprecogs, personas que pueden anular la capacidad de predicción del futuro de los precogs -estos últimos se daban cuenta de la presencia de los primeros porque su relación con el futuro se veía alterada-. La competencia entre uno y otros es está fuerte que existen dispositivos electrónicos para medir cuantos psis y antipsis hay en un lugar. El uso de estas personas es tan normal que en los periódicos se anuncia:

¿le sintoniza algún extraño, está realmente usted a solas, predice sus actos alguien a quien usted no conoce, que no querría conocer ni invitar a su casa? Termine su inquietud acudiendo a la organización de prevención más cercana, podrá saber si es víctima de una intrusión no autorizada, y siguiendo sus instrucciones, la organización cuidará de eliminar tal intrusión... a un precio muy asequible (Dick, 2012:15).

La vigilancia se construye así como una herramienta clave en las disputas entre grupos y personas en el espacio político y social. No es un procedimiento excepcional al que se recurre, sino una herramienta que normalmente se usa, ya sea para garantizar que la elección de una autoridad se respete o evitar que alguien modifique o intervenga en la conducta de otros. De esta manera la vigilancia queda naturalizada en la vida social como un procedimiento que forma parte de las relaciones sociales, un procedimiento que se encuentra inscrito en la manera en como los individuos se relacionan, que se mezcla con otras acciones y comportamientos. Sin embargo, eso no impide a Dick mostrar lo violentas que pueden ser las relaciones acompasadas por los procesos de vigilancia, ni 
señalar la manera en cómo destruyen identidades, colectivos y formas específicas de organización política.

\section{Paradojas de la vigilancia}

Los tipos de vigilancia que aparecen en las novelas aquí analizadas se presentan regularmente ligadas a otro tipo de dispositivos de monitoreo. Por ejemplo, en Fluyan mis lágrimas, dijo el policía, existe un aparato de alta demanda en el mercado, denominado "red transex", que funciona como un sistema de comunicación que vincula las fantasías sexuales de sus usuarios: los "aspectos sexuales de las personas conectadas son entrelazados electrónicamente entre sí [...] y amplificados, tanto como puedas soportar. Es algo adictivo porque es magnificado electrónicamente" (Dick, 1974: 176). Por su parte, en ¿Sueñan los androides con ovejas eléctricas?, se describe la operación de un dispositivo de vigilancia para monitorear el sentimiento de los humanos a través de un "climatizador de ánimo": una especie de aire acondicionado de las emociones humanas que regula la depresión y la apatía. Como Freedman (2000) sugiere, este tipo de tecnologías parecen estar en consonancia con los trabajos de corte foucaultiano, donde la vigilancia se dibuja como un proceso que disciplina, modela y gubernamentaliza. No obstante, a diferencia de la perspectiva cripto-normativa de Foucault -donde la vigilancia se dibuja como un proceso progresivo irreversible, que tiende a poner a cada sujetos bajo la lógica del control (Reed, 2011)-, la vigilancia tiene efectos contingentes e inesperados en las novelas de Dick, en la medida en que no necesariamente es un mecanismo de pura dominación (Deibert, 2003; Zedner, 2007). Su crítica, pero también su uso bajo principios civiles, puede terminar por expandir los principios y valores de la libertad, la autonomía y la individualidad. 
La política de identificación en ¿Sueñan los androides con ovejas eléctricas? propicia, por ejemplo, un temor generalizado entre la población a ser considerado un androide. En consecuencia, se genera un sentimiento de rechazo a dudar de la humanidad de las persona y, por tanto, al uso del test de Voigt-Kampff. Como sugiere Afonso da Silva (2007), Muri (2003) y Wald (2015), la permanente exigencia en la novela de corroborar la humanidad de cualquiera, tiene por consecuencia la reivindicación del carácter humano no sólo de los androides sino, paradójicamente, de los propios humanos. Por otro lado, cuando la policía queda desbordada por la información que generan los dispositivos de vigilancia -como sucede en Fluyan mis lágrimas, dijo el policía- la propia autoridad renuncia a vigilar a la población: el excesivo control colapsa por sí mismo, lo cual permite la emergencia de espacios de autonomía personal y colectiva. Una situación similar aparece en Una Mirada en la Oscuridad: al momento que la información que arrojan los holoescáneres fracasa al identificar a cualquier ciudadano, se generan dudas sobre la capacidad de vigilancia de estos dispositivos. Como plantean acertadamente Seed (2011) y Higgins (2015), la sobreproducción de información por parte de los sistemas de vigilancia, en varias de las novelas de Dick, hace dudar a sus operadores sobre lo que es en verdad la realidad.

Por lo que se refiere a la relación entre vigilancia y gestión de los deseos sociales cabe seńalar que, como se pudo observar en las novelas aquí revisadas, tanto las élites políticas como los grupos políticos revolucionarios tienen en la vigilancia uno de sus principales instrumentos para conocer la opinión pública e influir en ella. Si bien es cierto tanto gobernantes como rebeldes tratan de adecuarse y controlar a la sociedad, no lo hacen a la manera de la programación de los sentimientos o al lavado de cerebro característico de las novelas distópicas del periodo de entre guerras. De hecho, la propia vigilancia de las pasiones permite que las personas 
puedan acceder a una cierta autonomía. Esto se puede observar en el caso particular de Simulacra: aquellos que han escapado a Marte instalan sus propios androides orgánicos cerca de ellos para que los vigilen y puedan programar terapias que les permitan sobrevivir en el aislamiento. En este sentido, la vigilancia aparece a veces como una herramienta de gobierno, pero otras veces como un instrumento de autogobierno (Link, 2010; Gates, 2009). ${ }^{11}$

La presencia de la vigilancia como una mediación para la dominación y la resistencia política adquiere su perfil más definido en los escenarios de disputa entre grupos y personas que Dick construye en Lotería solar y Ubik. En estas narraciones Dick describe una vigilancia que opera desde distintas instancias, lo cual contrasta con el omnipresente y monolítico "Gran Hermano" orwelliano (Shapiro, 2009). La vigilancia se dibuja como multidireccional, descentralizada y heterogénea y no como un instrumento de control unidireccional, centralizado y homogéneo (Sheldon, 2015). A veces la vigilancia sirve al Estado, otras a las corporaciones, pero también ayuda a la resistencia, la revuelta y la construcción de la agencia moral de los actores (Srinivas, 1999; Bould, 2015). De esta manera, la visión de Dick, como sugiere Best y Kellner (2003), es apocalíptica pero no carente de una cierta esperanza irónica. Considero en este sentido que la vigilancia para Dick es un proceso que genera relaciones de dominación y dinámicas profundamente inciviles, pero otras veces, y no pocas, es un medio para la resistencia frente al poder; por tanto, hay detrás de ella valores

${ }^{11}$ Cabe señalar en este punto que la regulación de las emociones y los estados de ánimo, así como de los deseos, son un tema recurrente en la obra de Dick. En una novela como La invasión divina (1981), por traer a cuenta un ejemplo, se muestra un sistema de inteligencia artificial, llamado "Gran Fideo", que analiza las necesidades emocionales de los humanos. A través de una cantante, Linda Fox -que es un sistema de inteligencia artificial-, se difunde "un sonido creado por un equipo electrónico, un equipo eléctrico muy sofisticado" (Dick, 2013: 94)" para satisfacer las ansiedades humanas. 
y principios civiles. Por ello la vigilancia en la obra de Dick abre, parafraseando a Jameson (1988), un debate alrededor de un tema que parece ocupar la agenda política y social de nuestro tiempo de forma creciente, propiciando una reflexión propositiva sobre su función, su poder y sus límites estructurales. ${ }^{12}$

Como algunos autores han señalado, la vigilancia no puede entenderse en todos los casos como un proceso unidireccional bajo el control de una persona o institución (Lyon, 1994). Incluso cuando existen procesos de coordinación entre instituciones que tienen a su cargo distintos mecanismos de vigilancia, dicha coordinación es por lo regular desigual, desarticulada y heterogénea (Haggerty y Ericcson, 2000). A esto hay que sumar que la vigilancia en todas sus formas tiene que enfrentar las capacidades individuales y grupales que la confrontan (Bogard, 2006). La sociedad desarrolla, por ejemplo, experiencias que generan procesos de vigilancia desde abajo (sousviellance) hacia los poderes políticos y corporativos, lo que invierte la tradicional idea de que la vigilancia solamente se despliega desde espacios institucionales de poder. Hay también estrategias de invisibilidad (Gilliom y Monahan, 2012) y, por supuesto, redes de resistencia (Martin, Brakel y Bernhard, 2009).

Como bien sugiere Koskela (2006), hay que considerar además que la vigilancia permite recoger y difundir información, expresar ideas, así como prácticas corporales que se construyen en la privacidad y tiene por objetivo la esfera pública (Yar, 2003; Baz y Bruno, 2003). Por esta razón autores como Bauman y Lyon (2013) han planteado que la vigilancia en las redes sociales puede ser vista, para bien y para mal, como una especie de confesionario electró-

${ }^{12}$ Sólo hay que dar cuenta del incremento de las cámaras de vigilancia en los espacios urbanos a escala global, la tendencia mundial a establecer cartas de identidad nacional, la expansión de drones para el monitoreo de fronteras y para el desarrollo de la guerra a distancia, además de la consolidación de los dispositivos biométricos de control de la población. 
nico donde el mundo privado conquista lo público. No sólo eso, puede transformarse en un medio de cuidado entre pares -amigos, familiares y seres queridos- generando lo que Andrejevic (2005) ha denominado como vigilancia lateral u horizontal. En términos lúdicos, la instalación de video cámaras, por ejemplo, en espacio públicos y privados no sólo sirve para el cuidado y control social sino que, como algunos trabajos han mostrado (Mäkinen, 2016; Albrechtslund y Dubbeld, 2005), garantizan dinámicas de participación colectiva donde el juego es un elemento central.

\section{Reflexión final}

Los estudios sobre la vigilancia en ciencias sociales han usado de manera recurrente la figura del "Gran Hermano" para mostrar la fuerza que pueden llegar a adquirir las videocámaras en los espacios urbanos, los sistemas de espionaje en Internet y en las redes sociales, así como los procesos de monitoreo a los que son sometidas las personas en sus actividades laborales y de ocio tanto en el ámbito público y privado. La referencia de esa figura advierte no sólo sobre los efectos del escrutinio social, sino que proporciona un pathos en torno a los peligros a los que se enfrentan individuos y colectivos: el deterioro en su privacidad, la manera en como son clasificados, los procesos de inclusión y exclusión social a los que son sometidos. No obstante, la figura del "Gran Hermano" dificulta apreciar la complejidad sobre la que se definen las lógicas de la vigilancia en nuestros días. La expansión de la vigilancia en sociedades democráticas y altamente diferenciadas hace que aquella adquiera particularidades específicas.

La vigilancia puede entenderse, por tanto, como un proceso que construye lazos de cohesión y no sólo de coerción. El trabajo narrativo de Dick parece que logra dar cuenta de la diversidad y complejidad que entrañan las relaciones de vigilancia en la socie- 
dad. En las novelas que aquí se han revisado se proyectan modelos de sociedad en los que se pone en tensión los temores a la vigilancia en relación con sus efectos negativos en la libertad, la individualidad, la autonomía y la solidaridad. Valores que paradójicamente pueden llegar también a ser reivindicados utilizando dispositivos de vigilancia. La vigilancia apela a nuestros temores más profundos sobre el desarrollo de la modernidad, pero también a los valores positivos que la constituyen. Las novelas de Dick retratan las situaciones de la vigilancia de forma crítica y el lector puede identificarse con las tensiones que genera, ya sea porque la padezca en su experiencia cotidiana o porque contribuye en su desarrollo. Este autor logró en términos estéticos entender la vigilancia en más de un sentido. En su obra parece haber indicios para generar figuras y metáforas que condensen, de mejor manera que otras, la manera en cómo se construyen los espacios de libertad y control social, así como las dinámicas civil e inciviles de la vigilancia en la sociedad contemporánea.

\section{Bibliografía}

Afonso da Silva, Rafael, 2007, "Blade Runner: o humano na época de su a reproductibilidad e técnica", Tecnologia e Sociedade, vol. 3, núm. 4, pp. 181-204.

Albrechtslund, Anders y Lynsey Dubbeld, 2005, "The Plays and Arts of Surveillance: Studying, Surveillance as Entertainment", Surveillance \& Society, Kingston, vol. núms. 2-3, pp. 216-221

Alexander, Jeffrey, 2006, The Civil Sphere, University Press, Oxford. Alfaraz, Claudio, 2010, "Discursos de lo artificial. Blade Runner como representación de la técnica”, Tecnología y Conocimiento, vol. 7, núm. 3, pp. 45-59. 
Andrejevic, Mark, 2005, iSpy. Surveillance and Power in the Interactive Era, University Press of Kansas, Lawrence.

Arteaga, Nelson, 2015, "Latinoamérica y el apocalipsis: Iconos visuales en Blade Runner y Elysium", Revista Liminar, vol. 13, núm. 2, pp. 13-26.

Arteaga, Nelson, 2014, "Metamorfosis de la vigilancia: literatura y sociedad de 1984 a Neuromante", Culturales, vol. 2, núm. 1, pp. 161-186.

Barceló, Miquel, 2015, Ciencia Ficción. Nueva Guía de Lectura, Ediciones B, Barcelona.

Bauman, Zygmunt y David Lyon, 2013, Liquid Surveillance, Polity, Cambridge.

Best Stevens y Douglas Kellner, 2003, “The Apocalyptic Vision of Philip K. Dick", Cultural Studies $\nabla$ Critical Methodologies, Illinios, vol. 3, núm. 2, pp. 186-202.

Bogard, William, 2006, "Welcome to the society of control", en Surveillance and visibility, Toronto University Press, Toronto, pp. 4-25,

Booker, Keith, 1994, Dystopian Literature: A Theory and Research Guide, Greenwood Press, Westport.

Bould, Mark, 2015, “The futures market: American Utopias”, en The Cambridge Companion to American Science Fiction, Cambridge University Press, Cambridge, pp. 83-98.

Bradbury, Ray, 2005, Fahrenheit 451, Minotauro, México.

Brooks, Peter, 2005, Realist Vision, Yale University Press, New Haven.

Carrere, Emmanuel, 2004, Yo estoy vivo y vosotros estáis muertos. Philip K. Dick 1928-1982, Minotauro, Madrid.

Cohen, Stanley, 1985, Visions of Social Control, Polity Press, Cambridge. 
Deibert, Roland, 2003, "Black Code: Censorship, Surveillance, and the Militarization of Cyberspace", Journal of International Studies, Szczecin, vol. 32, núm. 3, pp. 501-530.

Dick, Philip K., 2005, The Collected Stories of Philip K. Dick, Citadel Press, New York.

, 2006, Una mirada a la oscuridad, (1977 1ra. Ed.), Minotauro, Barcelona, pp. 271.

, 2007, Lotería solar, (1955 1ra. Ed.), Minotauro, Barcelona.

, 2007, Simulacra, (1964 1ra. Ed.), Minotauro, Barcelona.

, 2007, Nuestros amigos de Frolik 8, (1970 1ra. Ed.), Minotauro, Barcelona.

, 2011, Fluyan mis lágrimas, dijo el policía, (1974 1ra. Ed.), Minotauro, Barcelona.

, 2013, Ubik, (1969 1ra. Ed.), Minotauro, Barcelona.

, 2013, La invasión divina, (1981 1ra. Ed.), Minotauro, Barcelona.

Freedman, Carl, 2000, Critical Theory and Science Fiction, Wesleyan University Press, Middletown.

García, Ricardo, 2006, “'Blade Runner’ o la pregunta por la dignidad humana”, Revista de Bioética y Derecho, vol. 4, núm. 6, pp. 1-3.

Gates, Kelly, 2009, "New Media Addiction”, Television \& New Media, Tulane, vol. 10 , núm. 1, pp. 58-60.

Gilliom John y Torin Monahan, 2012, Supervision: An introduction to the surveillance society, The University of Chicago Press, Chicago.

Habermas, Jürgen, 1989, The Structural Transformation of the Public Sphere: An Inquiry into a Category of Bourgeois Society, Cambridge, MIT Press. 
Haggerty Kevin y Richar Ericcson, 2000, "The surveillant assemblage”, British Journal of Sociology, vol. 51, núm. 4, pp. 605622.

Hartley, Leslie Poles, 1987, Facial Justice, Oxford Paperbacks, Oxford.

Hayles, Kahterin, 1997, "RFID: Human Agency and Meaning in Information-Intensive Environments", Theory, Culture \& Society, London, vol. 26, núms. 2-3, pp. 47-72.

Higgins, David, 2015, “American Science Fiction after 9/11”, en The Cambridge Companion to American Science Fiction, Cambridge University Press, Cambridge, pp. 44-57.

Huxley, Aldous, 2010, A brave new world, Harper Perennial, New York.

Jameson, Fredric, 2007, Archaeologies of the future. The desire called Utopia and other Science Fictions, Verso, New York.

Jameson, Fredric, 1989, Documentos de cultura. Documentos de barbarie. La narrativa como acto socialmente simbólico, Visor, Madrid.

Jameson, Fredric, 1988, The Ideologies of Theory. Essays 1971-1986, vol. 2: The Syntax of History, Routledge, London.

Kammerer, Dietmar, 2012, "Surveillance in Literature, Film and Television", en Routledge Handbook of Surveillance Studies, Routledge, New York, pp. 99-106.

Koskela, Hille, 2006, "The other side of surveillance: Webcams, power and agency", en Theorizing Surveillance: The Panoptic on and Beyond, Willan Publishing, Cullompton, pp. 163-181.

Lee, Gwen y Doris Sauter, 2000, What if our world is their heaven? The final conversations of Philip K. Dick, Overlook, New York.

Lem, Stanislaw, 1975, "Science Fiction: A hopeless case-with exceptions", en Philip K. Dick: Electric shepherd, Norstrilia Press, Melbourne, pp. 69-94. 
Levin, Ira, 2010, This Perfect Day, Pegasus Books, Nueva York.

Link, Carl, 2010, Understanding Philip K. Dick, University of South Carolina, South Carolina.

Link, Carl y Gerry Canavan, 2015, The Cambridge Companion to American Science Fiction, Cambridge University Press, Cambridge.

Lyon, David,1994, The Electronic Eye: The Rise of Surveillance Society, University of Minnesota Press, Minneapolis.

Mackey, Douglas, 1988, Philip K Dick, T Wayne, Boston.

Mäkinen, Liisa, 2016, "Surveillance On/Off: Examining Home Surveillance Systems From The User's Perspective", Surveillance \& Society, Kingston, vol. 14, núm. 1, pp. 59-77.

Martin, Aaron, Rosamunde Brakel y Daniel Bernhard, 2009, "Understanding resistance to digital surveillance Towards a multidisciplinary, multi-actor framework", Surveillance \& Society, Kingston, vol. 6, núm. 3, pp. 213-232.

Marks, Peter, 2005, "Imagining Surveillance: Utopian Visions and Surveillance Studies", Surveillance \& Society, Kingston, vol. 3, núms. 2-3, pp. 222-239,

Marx, Gary, 2009, "Soul Train: The New Surveillance in Popular Music", en Lessons from the Identity Trial: Anonymity, Privacy and identity in a Networked Society, Oxford University Press, Oxford, pp. 377-399.

Muri, Allison, 2003, "Of Shit and the Soul: Tropes of Cybernetic Disembodiment in Contemporary Culture", Body \& Society, London, vol. 9, núm. 3, pp. 73-92.

Nellis, Mike, 2009, "Since 'Nineteen Eighty Four': Representation of Surveillance in Literary Fiction", en New Directions in Surveillance and Privacy, Willan Publishing, Cullompton, pp. 178-204. 
Nolan, William F. y Johnson Clayton, 1969, Logan's Run, Dell Books, New York.

Novell, Noemí, 2009, "Vigilancia extrema y justicia preventiva: narración y espectacularidad en Minority Report", Formats: revista de comunicación audiovisual, vol. 9, núm. 5, pp. 21-29.

Nussbaum, Martha, 1990, Love's Knowledge: Essays on Philosophy and Literature, Oxford University Press, New York.

Palmer, Christopher, 2005, "Philip K. Dick", en $A$ companion to Science Fiction, Blackwell, Malden, pp. 389-397.

Philmus, Robert,1991, "The two faces of Philip K. Dick", Science Fiction Studies, Greencastle, núm. 18, pp. 93-103.

Pia Lara, Maria, 1998, "Narrative Cultural Interweavings: Between Fact and Fiction", en Moral Textures: Feminist Narratives in the Public Sphere, Polity Press, Cambridge, pp. 92-104.

Rand, Ayn, 1999, Anthem, Plume, New York.

Reed, Isaac, 2011, Interpretation and Social Knowledge. On the use of Theory in the Human Sciences, The University of Chicago Press, Chicago.

Rorty, Richard, 1989, Contingency, Irony, and Solidarity, Cambridge University Press, Cambridge.

Seed, David, 2011, Science Fiction, A very Short introduction, Oxford University Press, Oxford.

Shapiro, Michael, 2009, "Managing Urban Security: City Walls and Urban Metis", Security Dialogue, Ottawa, vol. 40, núms. 4-5, pp. 443-461.

Sheldon, Rebekah, 2015, "After America", en The Cambridge Companion to American Science Fiction, Cambridge University Press, Cambridge, pp. 206-218. 
Srinivas, Nidhi, 1999, "Managers as androids: Reading moral agency in Philip K. Dick”, Organization, vol. 6, núm. 4, pp. 609-624.

Sutin, Lawrence, 1989, Divine Invasions: A life of Philip K. Dick, Carroll \& Graf, New York, pp. 368.

Vaz, Paulo y Bruno, Fernanda, 2003, "Types of Self-Surveillance: from abnormality to individuals 'at risk' ", Surveillance \& Society, Kingston, vol. 1, núm. 3, pp. 272-291.

Vizcarra, Fernando, 2011, "Modernidades múltiples y perfiles identitarios en Blade Runner. Un ejercicio de análisis textual cinematográfico", Culturales, vol. 7, núm. 13), pp. 31-62.

Wald, Priscilla, 2015, "Science, technology, and the environment", en The Cambridge Companion to American Science Fiction, Cambridge University Press, Cambridge, pp. 179-193.

Whitebrook, Maureen, 1996, "Taking the narrative turn: what the novel has to offer political theory", en Literature and the Political Imagination, Routledge, London, pp. 32-52.

Williams Raymond, 2001, Cultura y sociedad, Nueva Visión, Buenos Aires.

Williams, Paul, 1986, Only apparently real, Arbor House, New York.

Yar, Majid, 2003, "Panoptic Power and the Pathologisation of Vision: Critical Reflections on the Foucauldian Thesis", Surveillance \& Society, Kingston, vol. 1, núm. 3, pp. 254-271.

Zamyantin, Yevgeny, 1993, We, Penguin Books, London.

Zedner, Lucia, 2007, "Pre-crime and post-criminology?", Theoretical Criminology, Oxford, vol. 11, núm. 2, pp. 261-281 y 1362-4806. 